\title{
HR 2142: A SEMI-DETACHED INTERACTING BINARY?
}

\author{
J. COTÉ, O. R. POLS \\ Astronomical Institute 'Anton Pannekoek', University of Amsterdam \\ Kruislaan 403, 1098 SJ Amsterdam, The Netherlands \\ Centre for High Energy Astrophysics, Amsterdam \\ L. B. F. M. WATERS \\ SRON Laboratory for Space Research Groningen \\ PO Box 800, 9700 AV Groningen, The Netherlands
}

\begin{abstract}
The energy distribution of HR 2142 is studied in order to test the hypothesis that this star is an interacting binary with a cool Roche-lobe filling companion. We find that, for any reasonable choice of $T_{\text {eff }}$, the companion should have been detected in the red part of the spectrum. We propose instead that the Be star is the outcome of a case B mass-transfer, which has pun it up. It is now surrounded by a mass-loss disc, rather than an accretion disc and is accompanied by either a helium star (resembling the $\phi$ Per system) or by a white dwarf. If the binary was born spinning rapidly, alternatively the companion may be a solar-type, unevolved main sequence star. In case of a Helium star companion, there may be Helium lines visible (like in $\phi$ Per), or the helium star may be detectable in the XUV, e.g. by the ROSAT XUV instrument. A white dwarf companion may be accreting material from the disc around the Be star and may show low luminosity $\mathrm{X}$-ray emission. This emission may have been seen in the ROSAT PSPC X-Ray Survey.
\end{abstract}

\section{Reference}

For a full account of this study we refer to: Waters, L.B.F.M., Coté, J., Pols, O.R. (1991) Astronomy and Astrophysics, in press 